\title{
Research of Energy Technological Parameters in the Processes of Heat Pump Utilization of Heat Exhaust Gases of Rotary Kilns
}

\author{
Petrash V.D., Baryshev V.P., Shevchenko L.F., Geraskina E.A. \\ Odessa State Academy of Civil Engineering and Architecture \\ Odessa, Ukraine
}

\begin{abstract}
This work is devoted to the study of energy technological parameters of heat pump utilization of exhaust gases heat from rotary kilns in production of building materials for heat technology and industrial heat supply. This goal is achieved due to the proposed new approach to the creation of energy-saving technology and improvement of the heat supply system based on the integration of contact-recuperative and thermal transformer heat recovery. The most important results are as following: a modified dependence of the conversion coefficient, an effective system for removing the waste gas heat for industrial and urban heat supply, a thorough assessment of the energy efficiency of the developed system, the dependence of the actual conversion coefficient on the energy technological parameters, as well as a rational degree of the exhaust gases pre-cooling, depending on their initial temperature and ratio of water consumption of the systems for technological and household purposes, and the range of their preferred ratio. The relationship between the consumption of the heating and heated medium in the process of the contact interaction has been substantiated. The results obtained differ from those existing by the complex accounting of the energy technological parameters in the analytical solution of the task. The practical significance of the results of the proposed technical solution lies in the fact that they can significantly reduce the consumption of the burned fuel due to a substantial expansion of the energy potential of the recovered heat, reduce heat, dust and gas emissions.

Keywords: heat pumps, thermal transformer, heat recovery, rotary kilns, energy efficiency.

DOI: https://doi.org/10.52254/1857-0070.2021.2-50.07

UDC: 621.036; 697.4
\end{abstract}

Investigarea parametrilor tehnologici energetici în procesele de utilizare a pompei de căldură a căldurii gazelor reziduale din cuptoarele rotative

Petraș V. D., Baryșev V. P., Şevchenko L. F., Geraskina E. A.

Academia de Stat din Odessa de Inginerie Civilă și Arhitectură, Odessa, Ucraina

Rezumat. Lucrarea este dedicată cercetărilor asupra parametrilor tehnologici şi energetici la utilizarea prin intermediul pompelor de căldură a căldurii reziduale a gazelor evacuate din cuptoarele rotative la producerea materialelor de construcție pentru ciclurile tehnologiilor de termoficare și aprovizionare industrială cu căldură. Obiectivul declarat este atins datorită argumentării teoretice și a elaborării unei abordări noi la crearea unei tehnologii de economisire a energiei și a sistemului de termoficare în baza integrării utilizării recuperative în contact și prin intermediul transformatorului termic a căldurii gazelor reziduale evacuate din cuptoarele rotative. Cele mai relevante rezultate sunt noile dependențe ale coeficientului de transformare, ce permit căutarea condițiilor raționale de funcționare pentru sistemul elaborat întru asigurarea transformării de înaltă eficiență a fluxurilor de energie a gazelor reziduale de la aprovizionarea cu căldură. În baza analizei rezultatelor cercetărilor analitice a fost efectuată estimarea complexă a eficienței energetice, determinată dependența coeficientului real de transformare în raport cu parametrii tehnologici și energetici, este identificat gradul preliminar de răcire rațională a gazelor îndepărtate, ce depind considerabil de temperatura inițială și de relația cererii de căldură a sistemelor de termoficare, ventilare și aprovizionare cu apă caldă de consum. Implementarea rezultatelor lucrării în producere asigură extinderea considerabilă a potențialului energetic la utilizarea căldurii gazelor reziduale, de asemenea, permite creșterea eficiențe de utilizare a energiei combustibilului primar și reducerea emisiilor de căldură, gaze și praf.

Cuvinte-cheie: pompe de căldură, transformator termic, recuperare de căldură, cuptoare rotative, eficiența energetica.

\section{Исследование энерготехнологических параметров в процессах теплонасосной утилизации} теплоты отработанных газов вращающихся печей

Петраш В. Д., Барышев В. П., Шевченко Л. Ф., Гераскина Э. А.

Одесская государственная академия строительства и архитектуры, Одесса, Украина

Аннотация. Работа посвящена исследованию энерготехнологических параметров теплонасосной утилизации теплоты отработанных газов вращающихся печей в производстве строительных материалов для

(C) Петраш В. Д., Барышев В. П.,

Шевченко Л. Ф., Гераскина Э. А., 2021 
теплотехнологического цикла и промышленного теплоснабжения. Поставленная цель достигается за счет теоретического обоснования и разработки нового подхода в создании энергосберегающей технологии и системы теплоснабжения на основе интеграции контактно-рекуперативной и термотрансформаторной утилизации теплоты отработанных газов вращающихся печей. Наиболее важными результатами являются новые зависимости коэффициента преобразования, позволяющие проводить поиск рациональных условий работы разработанной системы по обеспечению высокоэффективной трансформации энергетических потоков отработанных газов для теплоснабжения. В соответствии с поставленной целью разработана система, которая обеспечивает эффективный отбор утилизируемой теплоты из отработанных газов в процессе контактно-рекуперативного нагрева теплоносителя с трансформацией энергетических потоков для промышленного и коммунально-бытового теплоснабжения. На основе анализа результатов ее аналитического исследования проведена комплексная оценка энергетической эффективности, определена зависимость действительного коэффициента преобразования от энерготехнологических параметров, выявлена рациональная степень предварительного охлаждения отходящих газов, существенно зависящая от их начальной температуры и соотношения энергопотребностей систем отопления, вентиляции и горячего водоснабжения. Обоснована связь между расходами греющей и нагреваемой среды в процессе контактного взаимодействия. Установлен диапазон предпочтительного соотношения расходов воды технологического и коммунально-бытового назначения в системах теплопотребления. Полученные результаты отличаются от известных комплексным учетом энерготехнологических параметров в аналитическом решении поставленной задачи. Значимость полученных результатов состоит в том, что предлагаемое техническое решение на основе теплонасосной технологии позволяет существенно снизить расход топлива для промышленного и коммунально-бытового теплоснабжения. Внедрение результатов работы в производство обеспечивает существенное расширение энергетического потенциала утилизируемой теплоты отработанных газов, а также позволяет обеспечить повышение эффективности использования энергии первичного топлива и снижение тепловых и пылегазовых выбросов.

Ключевые слова: тепловые насосы, термотрансформатор, утилизация теплоты, вращающиеся печи, энергетическая эффективность.

\section{ВВЕДЕНИЕ}

Работа относится к области совершенствования энергетической эффективности теплотехнологического комплекса производства строительных материалов за счет расширения возможностей использования потенциала отработанных газов для промышленного теплоснабжения.

Существенным недостатком вращающихся печей в процессе производства строительных материалов, определяющим суть актуальных задач в решении общей проблемы энергосбережения, является крайне низкая эффективность использования энергии топлива (до 45\%) - потери теплоты с отработанными газами составляют $30 \ldots 35 \%$ [1].

При этом в стоимости конечной продукции топливная составляющая достигает $60 \%$. Отработанные газы, являющиеся наибольшим резервом вторичных энергоресурсов в технологическом процессе производства цемента и керамзита, обладают мощным теплоэнергетическим потенциалом, который в настоящее время практически не используется для производственного и коммунально-бытового теплоснабжения.

\section{АНАЛИЗ ЛИТЕРАТУРНЫХ ДАННЫХ И ПО- СТАНОВКА ПРОБЛЕМЫ}

На основе результатов исследования [2] автором обосновано, что установка контактного теплообменника для отработанных газов, снижает расход природного газа до 3\%. При этом контактный нагрев способствует очистке продуктов сгорания от оксидов азота, а нагретая вода контактным способом соответствует предъявляемым требованиям к ее качеству для промышленных предприятий.

В работе западных авторов [3] на основе математического моделирования исследовалась система теплоснабжения с центральным и вспомогательными тепловыми насосами, размещенными в структуре абонентских систем. Модель, которая учитывала температуру воды в сети и в системе низкотемпературного отопления и горячего водоснабжения, оценивала соответствующий коэффициент преобразования.

В [4] приведен анализ современных разработанных европейских систем теплоснабжения, на основе тепловых насосов с 
отражением возможных вариантов их дальнейшего развития.

В работе [5] приведен систематизированный обзор значительного количества разработанных систем западными учеными с применением тепловых насосов в низкотемпературных системах теплоснабжения.

На основе результатов аналитических исследований [6] проанализирована эффективность теплонасосной системы, использующей энергетический потенциал артезианской воды, а также воздушных потоков.

Авторами [7] проведен анализ теплонасосной системы водяного отопления с общим подходом к выбору на основе энергетических и экологических показателей.

В работе [8] характеризуется определяющее влияние температуры низкопотенциального источника энергии на эффективность ее преобразования и качество нагреваемой среды в финских зданиях с низким потреблением теплоты системами отопления.

Перспективным направлением энергосбережения является совершенствование парокомпрессионных систем теплоснабжения [9] с термодинамическими характеристиками рабочих тел в области критических температур, учитывающие неравномерность режимов отбора теплоты в процессе низкотемпературного нагрева воды.

В работе [10] рассматривается дополнительная возможность использования генерируемой теплоты на основе энергии интегрированных низкотемпературных источников в теплотехнологических процессах промышленного производства.

Результаты исследования предложенной системы [11], направленные на повышение энергетической эффективности систем теплоснабжения, предусматривают совершенствование процессов реверсивной выработки комбинированным агрегатом теплоты и холода в виде воды и воздуха для соответствующих абонентских систем.

В работах $[12,13]$ изложены обобщенные результаты разработок традиционных и теплонасосных технологий с экономической оценкой вклада соответствующих перспективных низкотемпературных источников в зданиях с минимальным энергопотреблением.

В предложенной теплонасосной системе [14] предусматривается нагрев воды для горячего водоснабжения в теплообменнике «труба в трубе» с использованием утилизируемой теплоты удаляемого воздуха в теплый период года в реверсивном режиме с одновременным охлаждением помещения.

В работах $[15,16]$ анализировалась система комбинированной выработки тепловой и электрической энергии с глубокой утилизацией теплоты продуктов сгорания с помощью парокомпрессионного теплового насоса. Интегрированная теплотехнологическая схема с теплонасосным контуром для отбора теплоты из отработанных газов системы мусоросжигательного комплекса (г. Мальмё) предусматривает логически обоснованный вариант использования энергии в замкнутом теплотехнологическом цикле очистки газов и водоподготовки для теплогенерирующей установки.

Для предложенной системы [17] контактно-рекуперативного отбора с парокомпрессионной утилизацией теплоты отработанных газов вращающихся печей для промышленного теплоснабжения установлена зависимость действительного коэффициента преобразования в виде модифицированного критерия комплексной оценки ее энергетической эффективности. Система обеспечивает высокие значения коэффициента преобразования при соответствующих соотношениях расходов греющей и нагреваемой среды в подсистемах технологического и коммунально-бытового теплоснабжения, учитывая соотношение расходов воды на отопительно-вентиляционные процессы и горячее водоснабжение.

Эффективность традиционной рекуперации теплоты отработанных газов вращающихся печей производства керамзита и цемента с температурой до $200^{\circ} \mathrm{C}$ с характерной остаточной запыленностью ограничивается сравнительно высокой исходной температурой энергоносителя, воспринимающего теплоту утилизации их охлаждения и конденсации содержащихся водяных паров. Теплонасосные технологии позволяют обеспечить охлаждение отработанных газов до предельно низкой и обоснованной температуры их охлаждения с повышением теплотехнологической и энергетической эффективности, а также надежности работы систем промышленного и коммунальнобытового теплоснабжения, в том числе и горячего водоснабжения.

Таким образом, анализ известных систем отбора теплоты из отработанных газов с по- 
зиции энергосбережения свидетельствует о необходимости поиска рациональных энерготехнологических параметров и методов более глубокого их охлаждения с одновременным повышением температурного уровня нагреваемой среды для абонентских систем. При этом в процессе охлаждения отработанных газов экономия теплоты позволит сократить до 1/3 энергии традиционно сжигаемого топлива.

\section{ЦЕЛЬ И ЗАДАЧИ ИССЛЕДОВАНИЯ}

Главной целью настоящего исследования является решение задачи комплексного определения рациональных условий для теплонасосной утилизации теплоты отработанных газов вращающихся печей для промышленного теплоснабжения. Изложенный подход открывает возможность повышения температурного потенциала абонентского теплоносителя, увеличения общего количества утилизируемой теплоты, а также защите окружающей среды за счет снижения теплового загрязнения и уменьшения количества вредных выбросов в атмосферу.

\section{ОПИСАНИЕ ПРИНЦИПИАЛЬНОЙ СХЕМЫ ТЕПЛОНАСОСНОЙ СИСТЕМЫ ТЕПЛОСНАБЖЕНИЯ}

Предложенный метод аналитического исследования реализуется в системе с патентной новизной [17], рис. 1, которая работает следующим образом. После традиционной очистки от пыли отработанные газы из вращающейся печи по каналу 2 поступают в фильтр тонкой очистки 26 , а затем направляются в рекуперативный теплообменник 12 для предварительного их охлаждения в «сухом» режиме. После этого газы поступают в первую контактную камеру 6, предложенной структуры, для предварительного охлаждения и увлажнения, а далее - во вторую 28 для более глубокого их охлаждения с возможностью нагрева воды до соответствующей значения температуры «мокрого термометра» в каждой из них. В первой камере 6 контактное взаимодействие потоков происходит при повышенной температуре воды после теплообменника 15, а во второй камере 28 обеспечивается более глубокое доохлаждение газов при температуре исходной холодной воды поступающей из трубопровода 17. Затем газы проходят рекуперативный теплообменник 7, в который поступает холодная вода с предельно низкой температурой после испарителя 22 парокомпрессионного контура термотрансформатора. В результате происходит глубокое охлаждение парогазовой смеси до $15 \ldots 20^{\circ} \mathrm{C}$ в режиме конденсации влаги с выделением соответствующей теплоты парообразования. Для предотвращения выпадения конденсата в дымовой трубе теплообменнике 8 обеспечивает незначительный нагрев охлажденного газа, после чего он выходит в атмосферу с температурой $20 \ldots 25^{\circ} C$.

Исходная холодная вода по трубопроводу 17 поступает во вторую камеру контактного взаимодействия 28 для более глубокого охлаждения газов. Часть потока холодной воды из трубопровода 17 по циркуляционному трубопроводу 25 после предварительного нагрева в рекуперативном теплообменнике 15 поступает в испаритель 22 контура термотрансформатора с рециркуляционным потоком подогретой воды после теплообменника 8. После теплообменника 15 поток подогретой воды по трубопроводу 25 поступает в испаритель 22 парокомпрессионного контура, на основе которого происходит дальнейший нагрев поступающей воды из поддона 5 в конденсаторном теплообменнике 21. Для повышения надежности работы контактных камер, так и всей системы, вода с повышенной концентрацией шлама удаляется шнековой системой 29 для последующего применения еe на начальной стадии замкнутого технологического цикла. После контактного взаимодействия с газом вода из поддона 5 циркуляционным насосом 10 подается в конденсаторный теплообменник 21, где происходит ее дальнейший термотрансформаторный нагрев. Здесь осуществляется догрев воды после контактного взаимодействия за счет отбираемой теплоты в испарителе 22 из воды, поступающей после теплообменников 8,13 и 15, а также теплового эквивалента приводной мощности компрессора 23.

В испарителе 22 теплоноситель охлаждается до предельно низкой температуры, обеспечивая высокоэффективную работу теплообменника 7 для охлаждения парогазовой смеси в режиме конденсации. После конденсатора вода с повышенной температурой разделяется на два параллельно работающих рециркуляционных контура. Первая часть общего потока, после прохождения через рекуператор предварительного 
охлаждения газов 12, направляется в теплообменник 1 с целью нагрева теплоносителя для отопительно-вентиляционных систем. После снижения температуры в теплооб- меннике 15 греющая вода поступает в распределитель 9 первой контактной камеры.

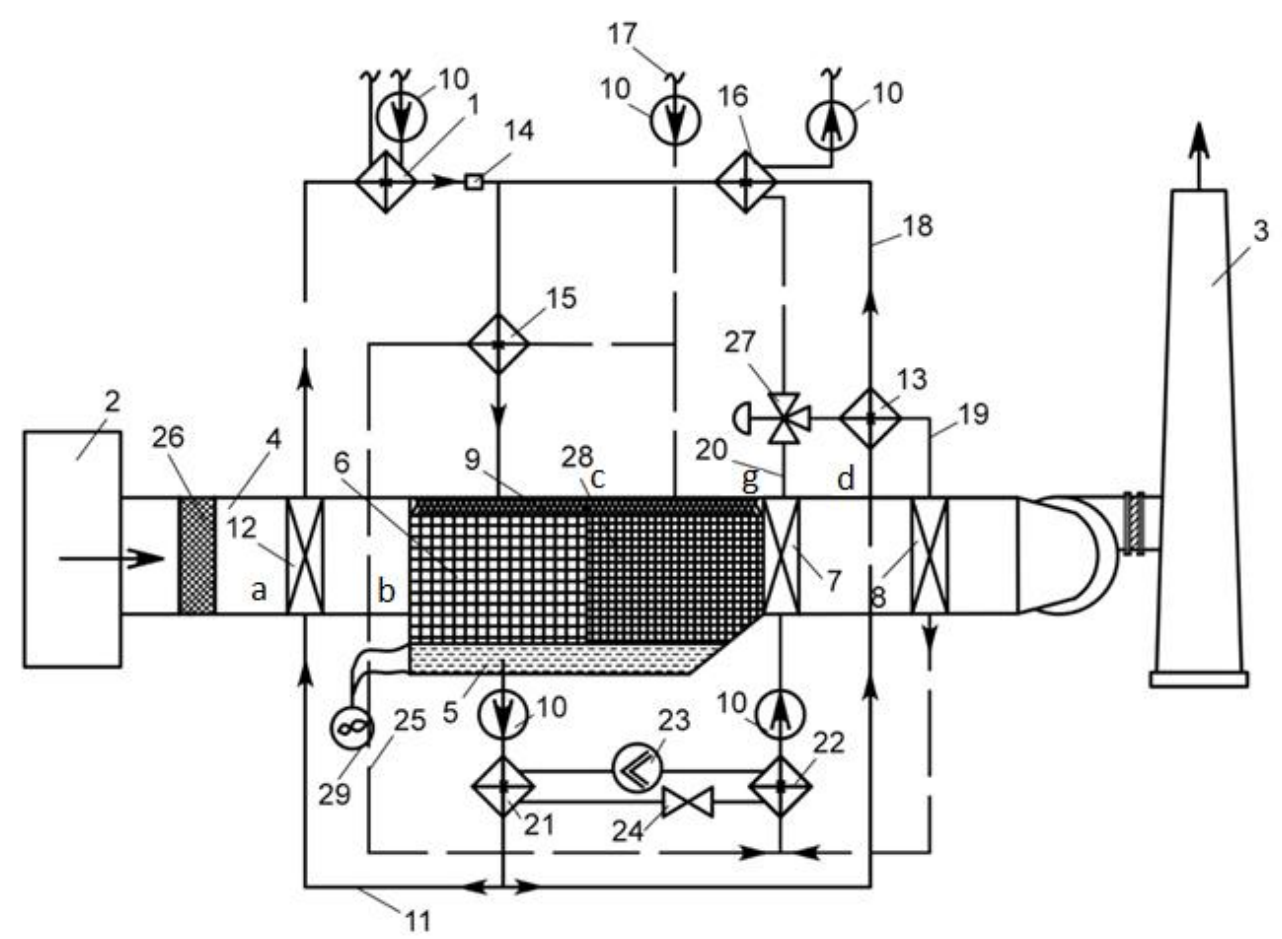

1 - теплообменник нагрева воды для отопительно-вентиляционных систем; 2 - газоход после традиционной очистки отработанных газов из вращающейся печи; 3 - дымовая труба; 4 - газоход после тонкой очистки отработанных газов; 5 - поддон для сбора воды в контактной камере; 6 - первая контактная камера предварительного увлажнения; 7 - теплообменник глубокого охлаждения газа; 8 - подогреватель газа перед дымовой трубой; 9 - ороситель; 10 - циркуляционные насосы; 11, 18, 19, 20 - трубопроводы; 12 - теплообменник предварительного охлаждения газов; 13, 15, 16 - теплообменники; 14 регулятор соотношения расходов; 17 - трубопровод подачи исходной холодной воды; 21 — конденсатор; 22 — испаритель; 23 - компрессор; 24 - дроссельный вентиль; 25 - трубопровод подачи воды на горячее водоснабжение; 26 - фильтр тонкой очистки; 27 — трехпозиционный регулятор расхода жидкости; 28 вторая контактная камера; 29 - дренажный участок технологического теплопотребления с регенерацией воды в поддоне 5.

Рис. 1. Схема системы контактно-рекуперативного отбора с трансформацией утилизируемой теплоты отработанных газов вращающихся печей для технологического и коммунально-бытового теплоснабжения. ${ }^{1}$

Вторая часть подогретой воды в конденсаторе 21, после теплообменников 13 и 16, поступает в тот же распределитель 9.

Охлажденная вода, после испарителя 22 термотрансформаторного контура, насосом 10 подается в теплообменник 7 для более глубокого охлаждения влагонасыщенных газов после второй контактной камеры. Частично нагретая вода в теплообменнике 7 , после прохождения по трубопроводу 20 с регулятором 27 , догревается в теплообменнике 16 и поступает в систему горячего во доснабжения. Рециркуляционная часть воды, после нагрева в теплообменнике 13 , по трубопроводу 19 поступает в теплообменник 8, а затем возвращается в испаритель 22 контура термотрансформатора.

\section{ТЕРМОДИНАМИЧЕСКИЙ АНАЛИЗ ТЕПЛООБМЕННЫХ ПРОЦЕССОВ}

Поступающий из вращающейся печи газ с начальной температурой $t_{g}$ с расходом сухой компоненты $G_{g}$ и пара $G_{v}$, нагревает 
воду в рекуперативном теплообменнике предварительного охлаждения 12.

При этом отбираемый тепловой поток $Q_{f t}$ в режиме «сухого» (без конденсации паров) охлаждения газа определяется по зависимости

$$
Q_{f t}=G_{g}\left[c_{g}\left(t_{g, a}-t_{g, b}\right)+d_{v}\left(c_{v, a} t_{v, a}-c_{v, b} t_{v, b}\right)\right]
$$

где $c_{g}$ и $c_{v}$ - средняя изобарная теплоёмкость газов и паров в рассматриваемых сечениях “ $a$ ” и “ $b$ ”, Дж/(кг К);

$t_{g}$ и $t_{v}$ начальная и конечная температура предварительного охлаждения газа и паров в соответствующих сечениях, ${ }^{\circ} \mathrm{C}$.

$d_{v}$ - влагосодержание газа, кг/кг.

В процессе последующего прохождения охлажденного газа через первую и вторую контактные камеры происходит нагрев воды. Общий тепловой поток охлаждения газа в контактной камере $Q_{c c}$ представляется в виде теплоты его «сухой» компоненты, пара и сконденсировавшейся его части

$$
\begin{array}{r}
Q_{c c}=G_{g}\left[\left(c_{g, b} t_{g, b}-c_{g, g} t_{g, g}\right)+\left(d_{v, b} i_{v, b}-\right.\right. \\
\left.\left.d_{v, g} i_{v, g}\right)+\left(d_{v, b}-d_{v, g}\right) c_{l} t_{l}\right], \mathrm{BT}
\end{array}
$$

где $c_{g}, c_{v}$ и $c_{l}$ - теплоёмкости соответственно «сухой» компоненты газа, паров и сконденсировавшейся части в сечениях " $b$ " и " $g$ " до и после контактной камеры, Дж/(кг·К);

$t_{g}, t_{v}$ и $t_{l}$ - температуры соответственно «сухой» компоненты газа, паров и сконденсировавшейся части в " $b$ " и " $g$ " до и после контактной камеры, ${ }^{\circ} C$;

$d_{v}$ - влагосодержание газа после контактной камеры, кг/кг;

$i_{g}, i_{v}$ - энтальпия газа и паров соответственно сухой компоненты газа, паров и сконденсировавшейся их части до и после контактной камеры, Дж/кг.

Тепловой поток доохлаждения газа после контактной камеры в теплообменнике 7 $Q_{h a c}$ определяется по зависимости

$$
\begin{aligned}
& Q_{h a c}=G_{g}\left[c_{g}\left(t_{g, g}-t_{g, d}\right)+\left(d_{v, g} i_{v, g}-\right.\right. \\
& \left.\left.d_{v, d} i_{v, d}\right)+\left(d_{v, g}-d_{v, d}\right) c_{l} t_{l}\right],
\end{aligned}
$$

где $c_{g}, c_{l}$ - теплоёмкость газа и сконденсировавшегося пара в соответствующих сечениях “" $g$ " и “ $d$ ”, Дж/(кг· $\mathrm{K})$;

$t_{g}, t_{l}$ - температура газа и сконденсировавшегося пара в сечениях " $g$ " и “ $d$ ", ${ }^{\circ} C$;

$d_{v}$ - влагосодержание пара в соответствующих сечениях, кг/кг.

Закономерность распределения нагреваемой воды в рассматриваемых подсистемах при взаимодействии ее с низкотемпературным газом определяется структурнофункциональной взаимосвязью основных элементов системы, рис. 1. Из балансного соотношения расходов следует, что расход воды во второй контактной камере $G_{c 2}=G_{c, w}-G_{h, w}$, а в первой $G_{c 1}=G_{v}$ Представив расход поступающий воды из поддона в исходный цикл технологического процесса $G_{\text {tech }}$ в виде $\Theta G_{v}$, получаем, что

$$
G_{\text {tech }}=\Theta G_{v}=G_{c, w}-G_{h, w}+G_{c o n d}, \mathrm{\kappa} \Gamma / \mathrm{c},(4)
$$

где $G_{c o n d}$ - общий расход паров, которые сконденсировались в контактной камере и доохладились в теплообменнике 7, кг/с.

Соотношение расходов воды в системах отопления $G_{\text {hew }}$ и горячего водоснабжения $G_{h w}$ можно представить в виде $\beta=G_{h e w} / G_{h, w}$.

Из соотношения расходов отработанного газа $G_{g}$ к общему расходу нагреваемой воды в первой $G_{c 1}$ и второй $G_{c 2}$ контактных камерах в виде

$$
a=G_{g} /\left(G_{c 1}+G_{c 2}\right)
$$

следует, что расход отработанного газа может быть представлен в более общем виде

$$
G_{g}=a\left(\beta G_{h, w}+G_{c, w}\right)
$$

Таким образом, тепловой поток нагрева воды в конденсаторе контура термотрансформатора $Q_{c}$ приобретает окончательный вид

$$
\begin{gathered}
Q_{c}=(1+\beta) G_{h, w} c_{l}\left\{t_{g}+\Delta t-\left(a / c_{l}\right)\left(1+G_{c, w} /\left(\beta G_{h, w}\right)\right)\right. \\
{\left[c_{g} \Delta t_{p c}^{*}\left(t_{g}-t_{e x}\right)+d_{v}\left(c_{v} t_{v}-c_{v, b} d_{v}\left(c_{v} t_{v}-\right.\right.\right.}
\end{gathered}
$$




$$
\left.\left(c_{v, b}\left(\left(t_{g}-\Delta t_{p c}^{*}\left(t_{g}-t_{e x}\right)\right)\right)\right]-t_{p}\right\}
$$

где $\Delta t$ - температурный перепад между конечными температурами греющей и нагреваемой среды в поверхностном теплообменнике, ${ }^{\circ} C$;

$\Delta t_{p c}^{*}$ - относительное снижение темпера-

туры газа в теплообменнике предварительного охлаждения, ${ }^{\circ} C$;

$t_{p}$ - температура воды в поддоне, ${ }^{\circ} C$. Ее значение было предварительно установлено аналитическим расчетом с анализом тепломассообменных процессов по диаграмме отработанных дымовых газов. В частности, вода в поддоне для газа с начальной температурой $(50 \ldots 100)^{\circ} \mathrm{C}$ находится в пределах $(27 \ldots 37)^{\circ} \mathrm{C}$;

$t_{e x}$ - температура уходящего газа, ${ }^{\circ} C$.

Для определения энергии охлаждения воды в испарителе контура термотрансформатора и газа после контактной камеры предварительно был установлен температурный потенциал теплоносителя для горячего водоснабжения в характерных точках системы.

На изложенной основе установлена зависимость для определения энергии охлаждения воды в испарителе и газа после контактной камеры.

В результате зависимость для определения энергии охлаждения циркулирующей воды через испаритель контура термотрансформатора приобретает следующий вид:

$$
\begin{gathered}
Q_{\mathrm{ev}}=G_{h, w} c_{l}\left\{\left[\left(G_{h, w} / G_{c, w}(1+\beta+j)+j\right)\right.\right. \\
Q_{\mathrm{ev}}=G_{h, w} c_{l}\left\{\left[\left(G_{h, w} / G_{c, w}(1+\beta+j)+j\right)\right.\right. \\
\left.\left.\left.\left(t_{g}-t_{e x}\right)-2 t_{g, d}+m t_{w, h}\right)\right)\right]\left[c _ { v } d _ { v } \left(t_{g}-(1+\mu)\right.\right. \\
\left.\left.\left(t_{g}-t_{e x}\right)-2 t_{g, d}+m t_{w, h}\right)-c_{l} t_{l}\left(d_{v, g}-d_{v, d}\right)\right]+ \\
\left.\left.G_{h, w} / G_{c, w}\left(\beta t_{r l}+t_{c}\right)\right]-(1+j) \mathrm{t}_{\mathrm{ev}, \mathrm{e}}\right\}(8)
\end{gathered}
$$

где $j$ - рециркуляционная часть общего расхода теплоносителя в системе горячего водоснабжения, проходящая через теплообменник 8 для предотвращения выпадения конденсата из охлаждённого газа при дальнейшем его контакте с поверхностями газоходов и выбросной трубы; $m \geq 1$ - коэффициент, отражающий степень приближения температуры нагрева воды к предельной температуре возможного насыщения газа водяными парами;

$t_{r l}$ - температура теплоносителя в обратной магистрали отопительно-вентиляционной системы, ${ }^{\circ} \mathrm{C}$;

$t_{w, h}$ - расчётная температура теплоносителя в системе горячего водоснабжения, ${ }^{\circ} C$;

$t_{e v, e}$ - конечная температура охлаждаемой воды после испарителя, значение которой в анализируемой системе логично рассматривать положительной в пределах $t_{e v, e}=4 \ldots 6^{\circ} \mathrm{C}$

Взаимосвязь расходов исходной холодной воды $G_{c w}$ и на горячее водоснабжение $G_{h, w}$ с учетом ранее принятого соотношения (6) $G_{\text {tech }}=\Theta G_{v}$ представляется в виде обобщенной взаимосвязи

$$
\mathrm{G}_{\mathrm{cw}}=G_{h, w}[1+\Theta(1+\beta)] 1,
$$

Воспринятый тепловой поток в конденсаторе $Q_{c}$ определяется тепловой мощностью испарителя $Q_{e v}$, а также тепловым эквивалентом приводной мощности компресcopa $W$ в процессе термотрансформации энергетических потоков, откуда следует, что $Q_{c}=Q_{e v}+W$. Исходя из определения действительного коэффициента преобразования и соответствующего соотношения энергетических потоков получаем, что

$$
\varphi=\left(1-Q_{e v} / Q_{c}\right)^{-1}
$$

После подстановки соответствующего соотношения тепловых потоков конденсатора $Q_{c}(7)$ и испарителя $Q_{e v}(8)$ в зависимость (10), эффективности преобразования энергии в анализируемой системе представляется возможным производить по следующему уравнению:

$$
\varphi=\left(1-\left(A B C^{-1}\right)\right)^{-1}
$$

где $A, B$ и $C$ - комплексы, учитывающие соответствующую взаимосвязь исходных и режимных параметров в испарителе и конденсаторе: 


$$
\begin{gathered}
A=(j+(1+\beta+j) /(1+\Theta(1+\beta))), \\
B=\left[t_{e v, e}+\frac{a}{(1+j) c_{l}}(1+\beta+\Theta(1+\beta))\right. \\
\left\{\left[t_{g}-(1+\mu)\left(t_{g}-t_{e x}\right)-2 t_{g, d}+m t_{w, h}\right]\right. \\
\left.\left(c_{g}+c_{v} d_{v}\right)-c_{l} t_{l}\left(d_{v, g}-d_{v, d}\right)\right\}+ \\
\left.\left(\beta t_{r l}+t_{w, c}\right) /(1+\Theta(1+\beta))\right]-(1+j) t_{e v, e}, \\
\mathrm{C}=(1+\beta+j)\left\{t_{g, e x}+\Delta t-\frac{a(1+\beta+\Theta(1+\beta))}{c_{l}(1+\beta+j)}\right. \\
{\left[c_{g} \Delta t_{p c}^{*}\left(t_{g}-t_{e x}\right)+d_{v}\left(c_{v} t_{v}-c_{v, b}\left(t_{g}-\right.\right.\right.} \\
\left.\left.\left.\Delta t_{p c}^{*}\left(t_{g}-t_{e x}\right)\right)\right)-t_{w}\right\} .
\end{gathered}
$$

Из уравнения обобщенной взаимосвязи (11) следует, что действительный коэффициент преобразования, как модифицированный критерий комплексной оценки эффективности трансформации энергетических потоков в предложенной системе, учитывающий ее исходные параметры и режимные условия работы структурных подсистем, зависит от сочетания выше рассмотренных факторов, и прежде всего от соотношения температур и расходов греющей и нагреваемой среды.

\section{АНАЛИЗ РЕЗУЛЬТАТОВ ИССЛЕДОВАНИЯ}

В практике инженерной разработки прежде всего представляет интерес влияния предварительного охлаждения отработанных газов на энергетическую эффективность термотрансформаторной системы теплоснабжения, что иллюстрируется на рис. $2^{1}$.

Из представленных графиков на рис. 2 следует, что рациональная степень предварительного охлаждения газов определяется, прежде всего, их начальной температурой. При этом целесообразные значения предварительного охлаждения отработанного газа с температурой $t_{g}=75^{\circ} \mathrm{C}$ находятся в пределах возрастания $\Delta t_{p c}^{*}=0,35 \ldots 0,5$ со снижением соотношения расходов энергоносителей $\beta$ в системах отопления и горячего водоснабжения в процессе эксплуатационного регулирования.

Для отработанных газов с более высокой температурой диапазон предварительного охлаждения газов $\Delta t_{p c}^{*}$ заметно сужается. Например, для газов с температурой $t_{g}=$ $150^{\circ} \mathrm{C}$ диапазон соответствующих значений значительно уменьшается и находится в пределах $\Delta t_{p c}^{*}=0,2 \ldots 0,25$ со снижением его начального значения. Здесь также отмечается аналогичная закономерность повышения эффективности преобразования энергетических потоков при снижении соотношения вышеуказанных расходов теплоносителя в процессе эксплуатационного регулирования.

Одним из основных факторов повышения энергетической эффективности работы анализируемой системы является зависимость коэффициента преобразования энергетических потоков согласно (11) от соотношения расходов а отработанного газа и поступающей воды в контактную камеру, которая иллюстрируется графически на рис. $3^{2}$.

Из графиков следует, что на коэффициент преобразования существенно влияет начальная температура отработанных газов и соотношение расходов воды в процессе эксплуатационного регулирования отопительно-вентиляционных систем и горячего водоснабжения. Очевидно, что достаточно высокие значение коэффициента преобразования до $10 \ldots 15$ обеспечиваются при соответствующих соотношениях анализируемых расходов $(a=0,2 \ldots 1,2)$. При более высоких значениях начальной температуры газов их смещение происходит при значениях значениям $a \leq 0,2$.

Диапазон рациональных соотношений отработанного газа и контактной воды, при которых обеспечивается высокоэффективная трансформация тепловых потоков, логично согласуется с обратной величиной коэффициента орошения, находящегося в пределах $0,5 \ldots 1,5$ кг/кг сухого воздуха, в охлаждающих системах контактного взаимодействия воды и газо-воздушных потоков, а также в системах кондиционирования.

Одним из определяющих параметров, обеспечивающих рациональные условия повышения энергетической эффективности системы с теплонасосной утилизацией является соотношение расходов воды на отопительно-вентиляционные процессы и горячее 


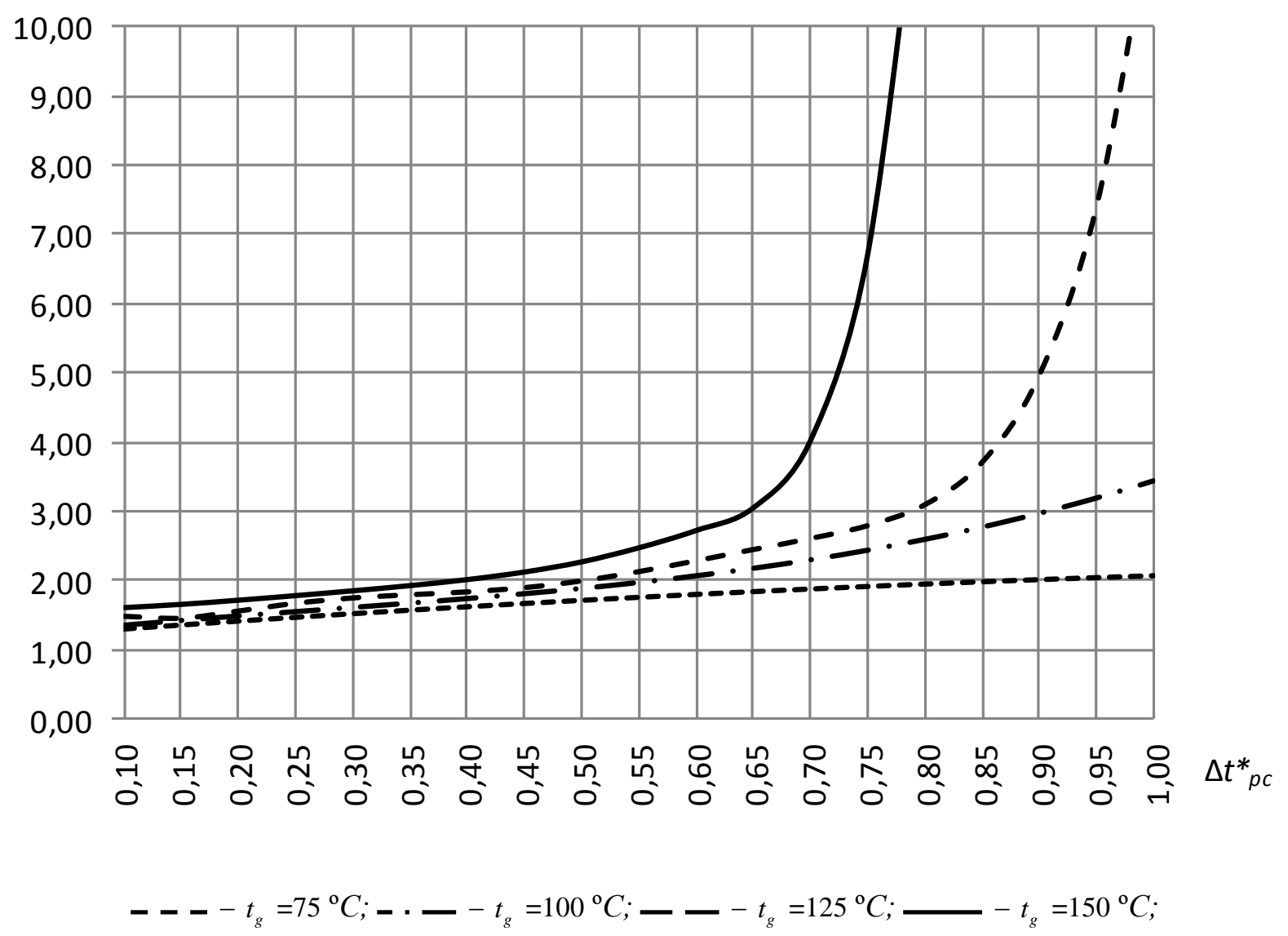

Рис. 2. Зависимость коэффициента преобразования от глубины предварительного охлаждения газа в рекуперативном теплообменнике, $\Delta t_{p c}^{*} \bullet^{2}$

Из представленных графиков на рис. 4, построенных на основе уравнения (11) следует, что высокая эффективность преобразования энергии в системе с температурой отходящих газов $100^{\circ} \mathrm{C}$ находится в диапазоне $\beta$ в пределах $0,3 \ldots 0,9$ для различных систем отопления с соответствующими расчетными перепадами температур теплоносителя.

Из этих графиков также следует, что эффективность преобразования энергетических потоков возрастает при соответствующем снижении расходов теплоносителя технологического и коммунально-бытового назначения. Из графиков следует целесообразность применения систем отопления с более низкими расчетными температурами теплоносителя, прежде всего низкотемпературных систем. Косвенно они также указывают на возрастающую эффективность преобразования энергии при снижении температур теплоносителя в процессе эксплуатационного регулировании с одновременным снижением соотношения теплоносителей технологического и коммунально-бытового назначения. Существенным фактором, влияющим на энергетическую эффективность системы, является предварительное охлаждение отработанных газов, которое позволяет обеспечить максимальный нагрев теплоносителя для отопительновентиляционных систем. Вместе с тем, температурный перепад предварительного охлаждения газов ограничивается нежелательным процессом возможной конденсации водяных паров при снижении их температуры в теплообменнике 12, что обусловливает ухудшение его теплообменных, аэродинамических и эксплуатационных характеристик.

Закономерно, что главным в анализируемом процессе должна быть рациональная степень предварительного охлаждения газов для обеспечения общей надежности работы при повышении энергетической эффективности предложенной контактнорекуперативной системы с трансформацией энергетических потоков. Представляет интерес анализ зависимости энергетической эффективности системы от соотношения 
расходов воды для технологического и коммунально-бытового теплоснабжения. Отбор нагретой воды из поддона контактной камеры является рациональным по энерготехнологическим показателям для использования еe на исходной стадии с температурой $45 \ldots 50^{\circ} \mathrm{C}$ в основном производственно-технологическом цикле.

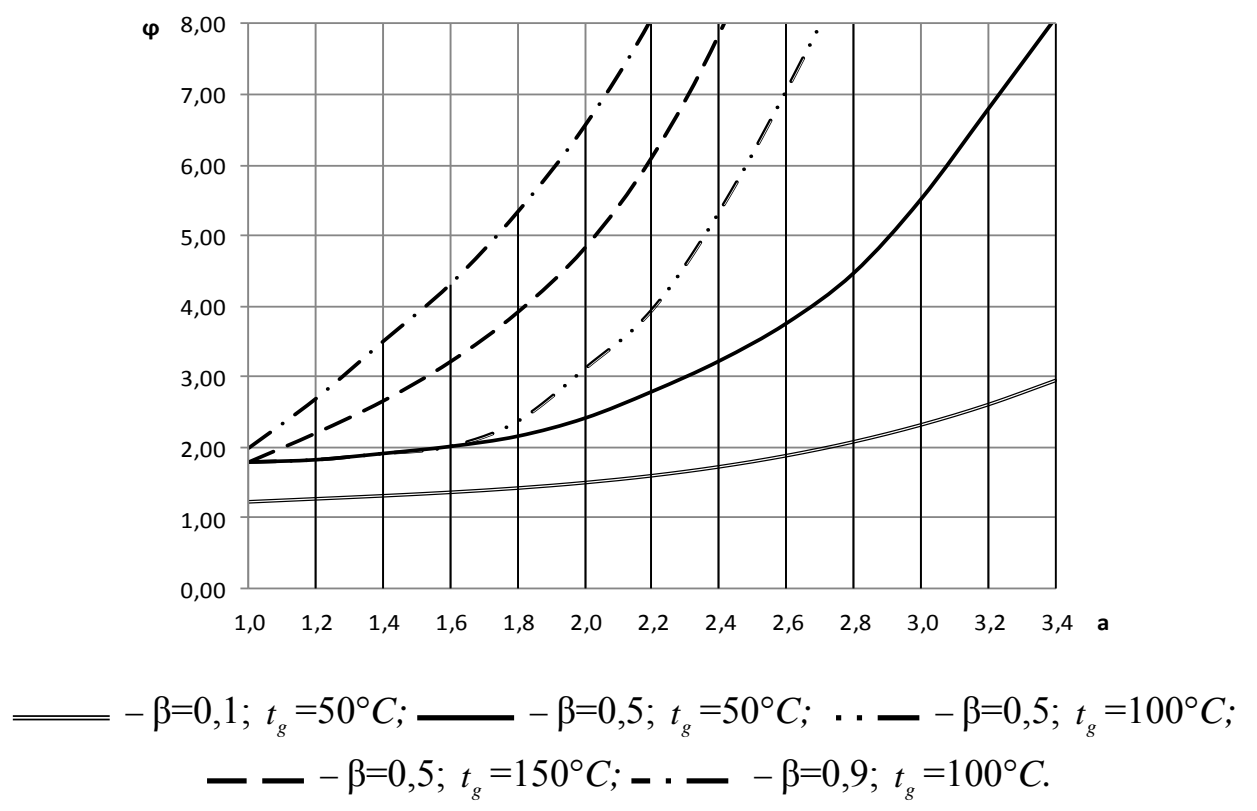

Рис. 3. Зависимость коэффициента преобразования от соотношения расходов отработанных газов и нагреваемой воды в контактной камере, $a .^{3}$

Кроме того, отбор воды обеспечивает надежную работу самой контактной камеры и соответствующих подсистем в результате непрерывной регенерации нагреваемой среды с остаточными пылегазовыми фракциями отработанного газа после его предварительной тонкой очистки.

На рис. 5. представлена зависимость коэффициента преобразования от соотношения расходов теплоносителей технологического и коммунально-бытового назначения при начальной температуре отработанных газов $100^{\circ} \mathrm{C}$ и $300^{\circ} \mathrm{C}$ с соответствующей степенью их предварительного охлаждения. $^{4}$

Из представленных графиков на рис. 5,a следует, что на эффективность преобразования существенно влияет начальная температура отработанных газов, а также степень их предварительного охлаждения в теплообменнике 12. Высокая эффективность преобразования энергетических потоков с коэффициентами $\varphi=10 \ldots 15$ при начальной температуре газов $100^{\circ} \mathrm{C}$ обеспечивается при соотношении расходов отбираемого теплоносителя для технологических потребностей и коммунально- бытового назначения в диапазоне $\Theta=0,3 \ldots 0,7$, учитывая рациональную степень предварительного охлаждения газового потока.

Вместе с тем при начальной температуре отработанных газов $300^{\circ} \mathrm{C}$, рис.5, b, рациональные значения указанного соотношения ограничивается диапазоном $0,25 \ldots 0,45$. На основе анализа результатов аналитического исследования проведена комплексная оценка энергетической эффективности утилизации теплоты отработанных газов вращающихся печей для промышленного теплоснабжения от исходных условий и режимных параметров предложенной системы. Установлено, что рациональное соотношение расходов воды для систем отопления с традиционными перепадами температур и горячего водоснабжения находится в пределах $0,3 \ldots 0,9$. При этом увеличение энергетической эффективности отмечается в процессе эксплуатационного регулирования систем со снижением соотношения расходов теплоносителей технологического и коммунально-бытового назначения.

Степень предварительного охлаждения отработанных газов, существенно зависящая от их начальной температуры, нахо- 
дится в пределах 0,35...0,5 при снижении соответствующих расходов теплоносителей в системах отопления и горячего водоснабжения. Рациональное соотношение расходов греющей и нагреваемой среды в про- цессе контактного взаимодействия, существенно зависящее от начальной температуры отработанных газов, находится в пределах $0,2 \ldots 1,2$.

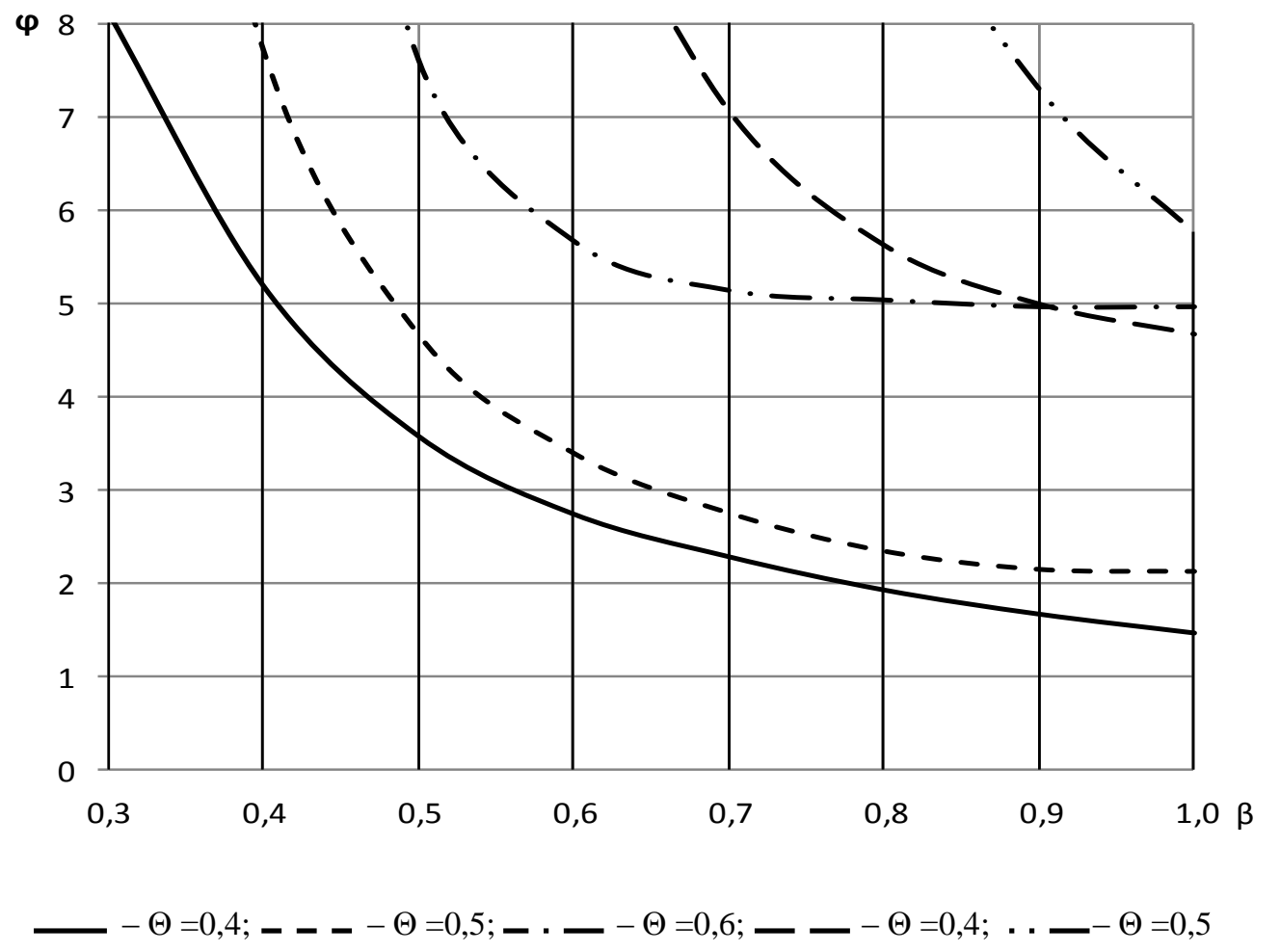

4. Зависимость коэффициента преобразования от соотношения расчетных параметров систем отопления и горячего водоснабжения, $\beta .{ }^{4}$

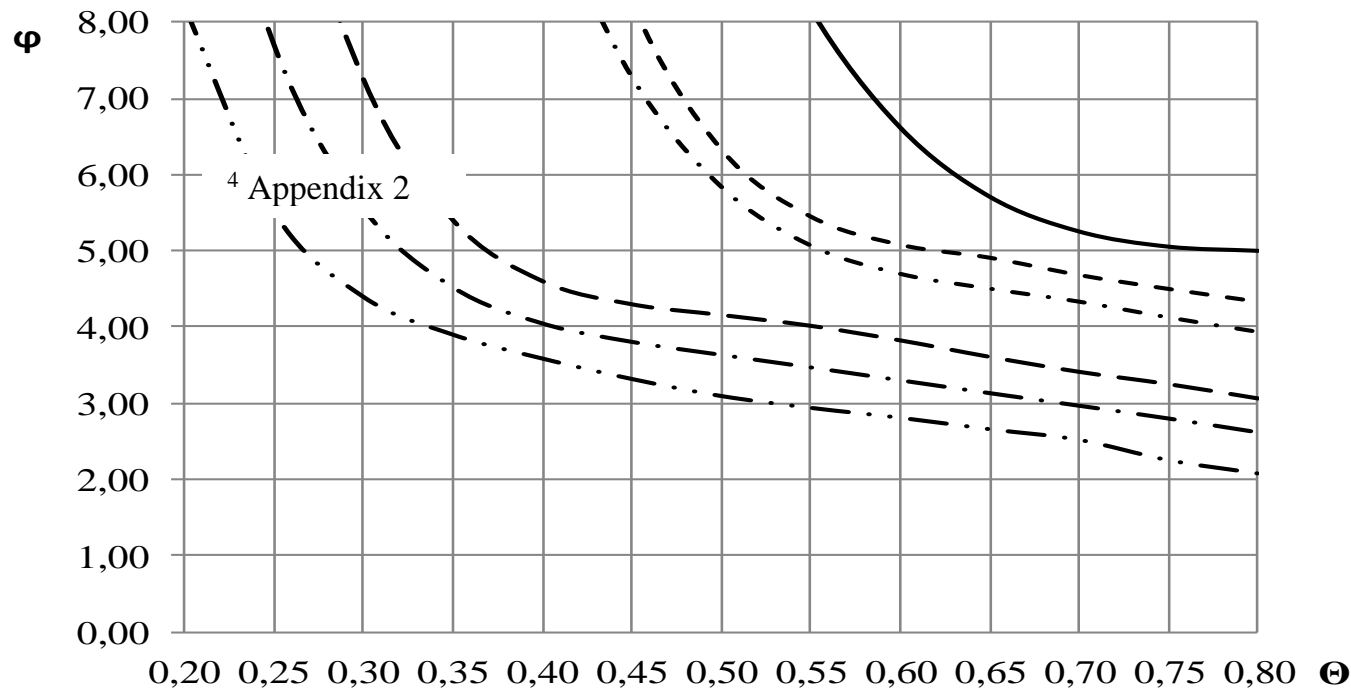

$\cdots--t_{g}=100^{\circ} \mathrm{C} ; \beta=0,9 ; \Delta t_{p c}^{*}=0,1 ;-\cdots--t_{g}=100^{\circ} \mathrm{C} ; \beta=0,5 ; \Delta t_{p c}^{*}=0,1 ;-{ }_{-}=100^{\circ} \mathrm{C} ; \beta=0,9 ; \Delta t_{p c}^{*}=0,2$;

- - - - $t_{g}=100^{\circ} \mathrm{C} ; \beta=0,5 ; \Delta t_{p c}^{*}=0,2 ;---t_{g}=100^{\circ} \mathrm{C} ; \beta=0,9 ; \Delta t_{p c}^{*}=0,3 ;-t_{g}=100^{\circ} \mathrm{C} ; \beta=0,5 ; \Delta t_{p c}^{*}=0,3$.

${ }^{4}$ Appendix 1 


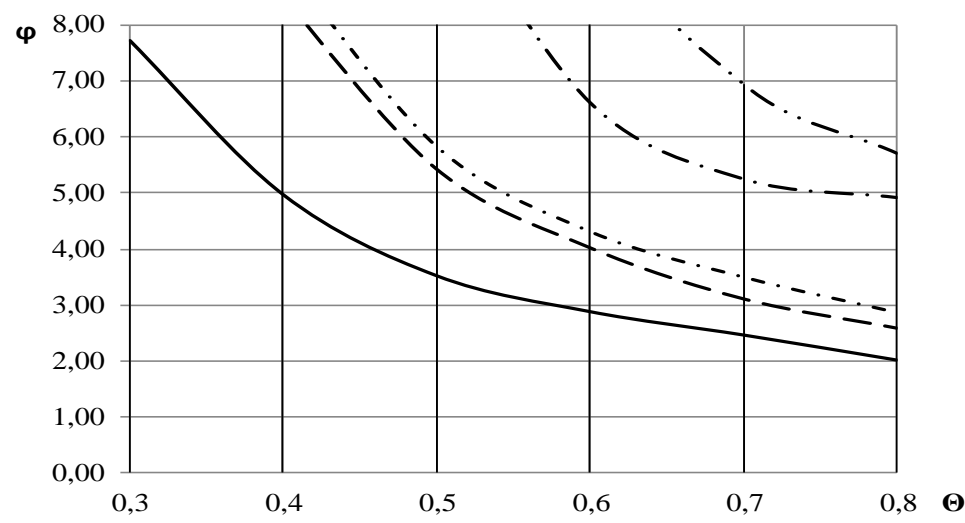

$-t_{g}=300^{\circ} \mathrm{C} ; \beta=0,9 ; \Delta t_{p c}^{*}=0,1 ;--t_{g}=300^{\circ} \mathrm{C} ; \beta=0,5 ; \Delta t_{p c}^{*}=0,1 ;-\cdots-t_{g}=300^{\circ} \mathrm{C} ; \beta=0,9 ; \Delta t_{p c}^{*}=0,2 ;$
$\cdots--t_{g}=300^{\circ} \mathrm{C} ; \beta=0,9 ; \Delta t_{p c}^{*}=0,3 ;-\cdots--t_{g}=300^{\circ} \mathrm{C} ; \beta=0,5 ; \Delta t_{p c}^{*}=0,2$

b)

Рис. 5. Зависимость коэффициента преобразования от соотношения расходуемой воды в системах технологического и коммунально-бытового теплоснабжение, $\Theta .^{5}$

Для отработанных низкотемпературных газов (до $50^{\circ} \mathrm{C}$ ) обратные значения анализируемого соотношения логично согласуются с соответствующими значениями коэффициента орошения.

\section{Выводы}

Предпочтительное соотношение расходов теплоносителей технологического и коммунально-бытового назначения, определяемое на основе установленных аналитических и графических зависимостей, находится в диапазоне $0,25 \ldots 0,7$ с обратно пропорциональным снижением его в зависимости от начальной температуры отработанных газов.

\section{APPENDIX 1 (ПРИЛОЖЕНИЕ 1)}

Fig. 1. Diagram of a contact-recuperative selection system with the transformation of the recovered heat of exhaust gases from rotary kilns for process and municipal heat supply: 1 - the water heat exchanger for heating and ventilation systems; 2 - the gas duct after traditional cleaning the waste gases from a rotary kiln; 3 - the chimney; 4 - the gas duct after fine cleaning the exhaust gases; 5 - the sump for collecting water in the contact chamber; 6 - the first contact chamber of preliminary humidification; 7 - the heat exchanger for deep gas cooling; 8 - the gas heater in front of the chimney; 9 - the sprinkler; 10 - the circulation pumps; $11,18,19,20$ - the pipelines; 12 - the heat exchanger for the gas precooling; $13,15,16$ - the heat exchangers; 14 -the regulator of the ratio of the heating agent flow rates; 17 - the initial cold water supply pipeline; 21 - the steam condenser; 22 - the evaporator; 23 - the compressor; 24 - the throttle valve; 25 - the hot water supply pipeline; 26 - the fine filter; 27 - the three-position fluid flow regulator; 28 - the second contact chamber; 29 - the drainage section of the technological heat consumption with water regeneration in sump 5.

Fig. 2. The dependence of the conversion factor on the depth of the pre-cooling gas in the recuperative heat exchanger $\Delta t_{p c}^{*}$.

Fig. 3. Dependence of the conversion factor on the ratio of the flow rates of exhaust gases and heated water in the contact chamber $a$.

4. Dependence of the conversion factor on the ratio of the calculated parameters of heating and hot water supply systems $\beta$.

Fig. 5. Dependence of the conversion factor on the ratio of consumed water in the systems of technological and municipal-household heat supply $\Theta$.

\section{APPENDIX 2 (ПРИЛОЖЕНИЕ 2)}

Исходные данные, использованные при построении графиков на рис. 2:

$$
\begin{aligned}
& j=0,2 ; t_{e v, e}=5^{\circ} C ; a=0,1 \ldots 2 ; \beta=0,1 ; 0,5 ; 0,9 ; \\
& \mu=0,04 ; t_{g}=75 ; 150^{\circ} \mathrm{C} ; t_{g 2}=20^{\circ} \mathrm{C} ; m=1 \text {; } \\
& \left.t_{w}=44^{\circ} C ; \quad c_{v}=1100 \text { Дж/(кг } \mathrm{K}\right) ; \\
& c_{l}=4200 Д ж /\left(\text { кг K); } c_{g}=1026 Д ж /(\text { кг } \mathrm{K}) ;\right. \\
& t_{l}=35^{\circ} C ; d_{v}=0,04 \kappa \Gamma / \mathrm{\kappa} ; d_{v, g}-d_{v, d}=0,02 \kappa \Gamma / \kappa \Gamma ; \\
& t_{e x}=25^{\circ} \mathrm{C} ; t_{r l}=70 ; 65^{\circ} \mathrm{C} ; t_{w, h}=55^{\circ} \mathrm{C} ; \Delta t=5^{\circ} \mathrm{C} \text {; } \\
& t_{g, e x}=95^{\circ} C ; \quad 85^{\circ} C ; \quad c_{e x}=1009 Д ж /(\text { кг } \mathrm{K}) ;
\end{aligned}
$$


$\Delta t_{p c}^{*}=0,1-0,5 ; \quad \Theta=0,5 ; \quad t_{v}=45 \ldots 75^{\circ} \mathrm{C} ;$ $c_{v, b}=1026$ Дж/(кг К).

Исходные данные, использованные при построении графиков на рис. 3 :

$j=0,2 ; t_{e v, e}=5^{\circ} C ; a=0,1 \ldots 2 ; \beta=0,1 ; 0,5 ; 0,9$;

$\mu=0,04 ; t_{g}=100 \ldots 300^{\circ} \mathrm{C} ; t_{g 2}=20^{\circ} \mathrm{C} ; m=1 ;$

$t_{w}=44^{\circ} C ; \quad c_{v}=1100 Д ж /(\kappa \Gamma \quad \mathrm{K}) ;$

$c_{l}=4200 Д ж /(\kappa \Gamma \cdot \mathrm{K}) ; \quad c_{g}=1026$ Дж/(кг К);

$t_{l}=35^{\circ} C ; d_{v}=0,04 \kappa \Gamma / \kappa г ; d_{v, g}-d_{v, d}=0,02 \kappa \Gamma / \kappa г ;$

$t_{e x}=25^{\circ} \mathrm{C} ; t_{r l}=70^{\circ} \mathrm{C} ; t_{w, h}=55^{\circ} \mathrm{C} ; \Delta t=5^{\circ} \mathrm{C}$;

$t_{g, e x}=95^{\circ} C ; \quad c_{v}=1100$ Дж/(кг $\left.\mathrm{K}\right) ;$

$c_{e x}=1009$ Дж/(кг К); $\Delta t_{p c}^{*}=0,2 ; \Theta=0,5 ;$

$t_{v}=100^{\circ} C ; c_{v, b}=1026$ Дж/(кг К $)$.

Исходные данные, использованные при построении графиков на рис. 4 :

$j=0,2 ; t_{e v, e}=5^{\circ} C ; a=1 ; \beta=0,1 \ldots 1 ; \mu=0,04 ;$

$t_{g}=50-150^{\circ} \mathrm{C} ; t_{g 2}=20^{\circ} \mathrm{C} ; m=1 ; t_{w}=44^{\circ} \mathrm{C}$;

$c_{v}=1100 Д ж /(\kappa \Gamma \cdot \mathrm{K}) ; c_{l}=4200 Д ж /(\kappa \Gamma \cdot \mathrm{K})$;

$c_{g}=1026 Д ж /(\kappa \Gamma \cdot \mathrm{K}) ; t_{l}=35^{\circ} C ; d_{v}=0,04 \kappa \Gamma / \kappa г ;$

$d_{v, g}-d_{v, d}=0,02$ кг/кг; $t_{e x}=25^{\circ} C ; t_{r l}=70^{\circ} C$;

$t_{w, h}=55^{\circ} \mathrm{C} ; \Delta t=5^{\circ} \mathrm{C} ; t_{g, e x}=95^{\circ} \mathrm{C}$;

$c_{e x}=1009 Д ж /(\kappa \Gamma \cdot \mathrm{K}) ; \Delta t_{p c}^{*}=0,1-0,3 ; \Theta=0,5 ;$

$t_{v}=100^{\circ} C ; c_{v, b}=1026$ Дж/(кг $\left.\mathrm{K}\right)$.

Исходные данные, использованные при построении графиков на рис. 5:

$j=0,2 ; \quad t_{e v, e}=5^{\circ} C ; \quad a=0,5 ; 1 ; 1,5$;

$\beta=0,9 ; 0,5 ; 0,7 ; \mu=0,04 ; t_{g}=100 ; 300^{\circ} \mathrm{C}$;

$t_{g 2}=20^{\circ} C ; m=1 ; t_{w}=44^{\circ} C ; c_{v}=1100 Д ж /(\kappa г ~ K) ;$

$c_{l}=4200 Д ж /(\kappa \Gamma \mathrm{K}) ; c_{g}=1026$ Дж/(кг К);

$t_{l}=35^{\circ} \mathrm{C} ; d_{v}=0,04 \kappa \Gamma / \kappa \Gamma ; d_{v, g}-d_{v, d}=0,02 \kappa \Gamma / \kappa г ;$

$t_{e x}=25^{\circ} \mathrm{C} ; t_{r l}=70^{\circ} \mathrm{C} ; t_{w, h}=55^{\circ} \mathrm{C} ; \Delta t=5^{\circ} \mathrm{C} ;$

$t_{g, e x}=95^{\circ} C ; \quad c_{v}=1100$ Дж/(кг $\left.\mathrm{K}\right) ;$

$c_{e x}=1009$ Дж/(кг $\left.\mathrm{K}\right) ; \Delta t_{a, b}=0,2^{\circ} C ; \Theta=0,5 ;$

$t_{v}=100^{\circ} C ; c_{v, b}=1026$ Дж/(кг К $)$.

\section{ЛИТЕРАTУРA (REFERENCES)}

[1] Lisienko V.G., Shchelokov Ya. M., Ladygichev M.G. Vrashchayushchiesya pechi: teplotekhnika, upravlenie i ekologiya [Rotary kilns: heat engineering, management and ecology]. Moskva, Teplotekhnik, 2004. 689 p.
[2] Zhikhar G.P., Zakrevskiy V.A. Ispol'zovanie teploty ukhodyashchikh gazov kotel'nykh agregatov $\mathrm{v}$ kontaktnom teploobmennike [Use of the heat of flue gases from boiler units in a contact heat exchanger]. Energetika [Energetics]. BNTU Minsk, 2010, p.p. 41-49. (In Russian).

[3] Østergaard P.A., Andersen A.N. Booster heat pumps and central heat pumps in district heating. Applied Energy, 2016, no. 184, p.p. 13741388.

[4] Sayegh M.A., Jadwiszczak P., Axcell B.P., Niemierka E., Brys K., Jouhara H. Heat pump placement, connection and operational modes in European district heating. Energy \& Buildings, 2018, no.166, p.p. 122-144.

[5] Ommen T.S. Heat Pumps in CHP Systems: High-efficiency Energy System Utilizing Combined Heat and Power and Heat Pumps. DTU Mechanical Engineering. DCAMM Special Report, 2015. no. S187.

[6] Pieper H., Ommen T., Elmegaard B., Markussen W.B. Assessment of a combination of three heat sources for heat pumps to supply district heating. Energy, 2019, no. 176, p.p. 156-170.

[7] Oussama Ibrahim, Farouk Fardoun, Rafic Younes, Hasna Louahlia-Gualous. Review of water-heating systems: General selection approach based on energy and environmental aspects. Building and Environment, 2014, vol. 72, p.p. 259-286.

[8] Rauno Holopainen, Kari Salmi, Erkki Kähkönen. Primary energy perfomance and perceived indoor environment quality in Finnish low energy and conventional houses. Building and Environment, 2015, vol 87, p.p. 92101.

[9] Kleefkens O., Spoelstra S. R\&d on industrial heat pumps. [11-th IEA Heat Pump Conference]. Montreal, 2014, p. 12-16.

[10] Atmaca I., Kocak S.Theoretical energy and exergy analyses of solar assisted heat pump space heating system. Thermal science, 2014, no 18 , pp. 417-427.

[11] Strategic Research and Innovation Agenda for Renewable Heating \& Cooling, European Technology Platform on Renewable Heating and Cooling. RHC-Platform, Brussels, 2013, p. 104.

[12] Murphy R. Integrated Heat Pump (IHP) System Development [Text]/ R. Murphy, C. Rice, V.Baxter // Oak Ridge, (Technical report), 2007, p. 45

[13]Economical heating and cooling systems for low energy houses. IEA Heat Pump Centre, Borås, Sweden, 2011, no. 32, p. 97.

[14] European Heat Pump Summit 15-16 October 2013. Available at: http://www.hpsummit.de/en. (accessed 19.10.2015). 
[15] Olsen J. Waste inceniration in Malmo// News from DBDH, 2000, no. 4, p.p. 28-30.

[16]Zhovmir M. M. Utilizatsiya nyz'kotemperaturnoi teploty produktov sgoraniya palyv za dopomogoyu teplovykh nasosiv [Utilization of low-temperature heat of combustion products of fuels by means of heat pumps]. Prom. teplotekhnyka [Industrial heat-

\section{Сведения об авторах}

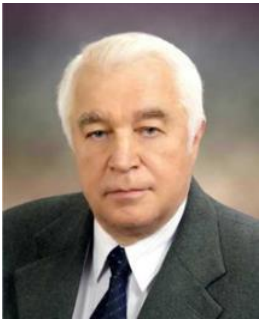

Петраш Виталий Демьянович, доктор технических наук, профессор. Область научных интересов: системы обеспечения микроклимата на основе энергии возобновляемых и вторичных источников, теплонасосные системы отопления и теплоснабжения, энергосбережение и экологически чистые технологии.

E-mail: petrant@ukr.net

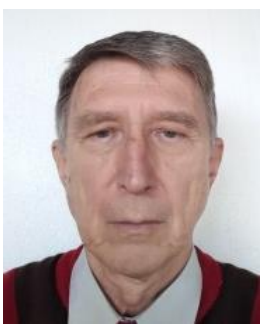

Барышев Виталий Павлович, кандидат технических наук, доцент по кафедре физики. Область научных интересов: энергетическая эффективность и экологически чистые технологии.

E-mail: baryshev@ogasa.org.ua ing technology], 2008, no. 32, p.p. 90-98. (in Ukrainian).

[17]Polunin Yu. N. e.a. Termotransformatorna sistema vidboru teploti $\mathrm{z}$ vidprats'ovanikh gaziv dlya promislovogo teplopostachannya [Thermotransformer system of heat extraction from exhaust gases for industrial heat supply]. Patent UA, no. 100923, 2013.

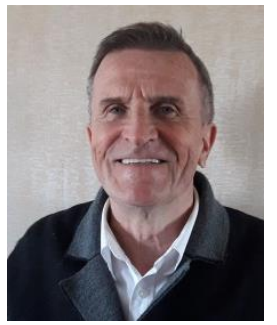

Шевченко Леонид Фёдорович, кандидат технических наук, доцент. Область научных интересов: системы обеспыливания технологических процессов, гелиосистемы горячего водоснабжения и энергетический аудит. E-mail: bgedyx7@ukr.net

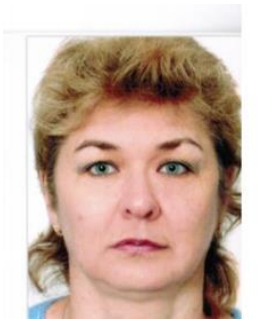

Гераскина

Элина

Анатольевна, к.т.н., доцент. Область научных интересов: использование энергии возобновляемых и вторичных источников, теплонасосные системы отопления и теплоснабжения, энергосбережение E-mail: poselok@te.net.ua 